\title{
Theoretical Evaluation of the Detectability of Random Lesions in Bayesian Emission Reconstruction ${ }^{\star}$
}

\author{
Jinyi Qi \\ Lawrence Berkeley National Laboratory, Berkeley, CA 94720, USA
}

\begin{abstract}
Detecting cancerous lesion is an important task in positron emission tomography (PET). Bayesian methods based on the maximum a posteriori principle (also called penalized maximum likelihood methods) have been developed to deal with the low signal to noise ratio in the emission data. Similar to the filter cut-off frequency in the filtered backprojection method, the prior parameters in Bayesian reconstruction control the resolution and noise trade-off and hence affect detectability of lesions in reconstructed images. Bayesian reconstructions are difficult to analyze because the resolution and noise properties are nonlinear and object-dependent. Most research has been based on Monte Carlo simulations, which are very time consuming. Building on the recent progress on the theoretical analysis of image properties of statistical reconstructions and the development of numerical observers, here we develop a theoretical approach for fast computation of lesion detectability in Bayesian reconstruction. The results can be used to choose the optimum hyperparameter for the maximum lesion detectability. New in this work is the use of theoretical expressions that explicitly model the statistical variation of the lesion and background without assuming that the object variation is (locally) stationary. The theoretical results are validated using Monte Carlo simulations. The comparisons show good agreement between the theoretical predications and the Monte Carlo results.
\end{abstract}

\section{Introduction}

Task-specific evaluation of medical imaging methods has become increasingly important. Two major applications of PET are lesion detection and region of interest quantitation. Due to the low signal to noise ratio (SNR) in PET data, statistically based image reconstruction methods have been developed to improve image quality [1-4]. To explore the full potential of statistical reconstruction, the reconstruction methods need to be optimized for PET applications. Such optimization requires fast computation of the task-specific figures of merit for statistical reconstructions. Here we focus on the detection task.

\footnotetext{
* This work is supported in part by the National Institute of Biomedical Imaging and Bioengineering under grants R01 EB00363, R01 EB00194, and by the Director, Office of Science, Office of Biological and Environmental Research, Medical Sciences Division, of the U.S. Department of Energy under contract no. DE-AC03-76SF00098.
} 
A general methodology for studying lesion detectability is the human observer ROC (receiver operating characteristics) study, where physicians read images and decide whether there is a lesion or not. A ROC curve is generated by plotting the true positive fraction vs. the false positive fraction. Using human observers can be time consuming, so numerical observers based on signal detection theory have been developed [5]. While numerical observers reduce the time of ROC studies, Monte Carlo reconstructions are often needed to provide the sample images. Hence the total computation time is still long.

While theoretical analysis is difficult because statistical algorithms are nonlinear, progress has been made in understanding the nonlinear properties of statistical reconstruction methods. Barrett et al. [6] derived approximate formulae for the mean and covariance of the maximum likelihood (ML) expectation maximization (EM) reconstruction as a function of the iteration number. The same approach was extended to the maximum a posteriori (MAP) EM algorithms by Wang and Gindi [7] and most recently to block iterative algorithms by Soares et al [8]. Using the results in [6] with numerical observer models, Abbey et al. [9] have studied lesion detectability in ML EM reconstruction. This iteration-based approach is attractive for methods that are terminated before convergence, as is common practice for the EM algorithm and its ordered-subsets variants [10]. However, evaluation of the expressions for large numbers of iterations is timeconsuming. In addition, this approach requires that the reconstruction algorithm has an explicit update equation. Hence, it is not applicable to gradient-type algorithms that involve line searches.

An alternative approach was proposed by Fessler and Rogers [11, 12], who analyzed the mean, variance, and spatial resolution at a fixed point of the objective function. The resolution and noise properties are computed at the fixed point using partial derivatives and truncated Taylor series approximations. These results are independent of the particular optimizing algorithm used and require only that the algorithm iterates to effective convergence. Qi and Leahy $[13,14]$ extended this approach by deriving simplified expressions for the local impulse response function and covariance using Fourier transform. These expressions allow fast evaluation of the resolution and noise properties of Bayesian reconstruction. The results have been used to choose the prior parameter to maximize the contrast to noise ratio [13] and to achieve uniform contrast recovery in fully 3D PET [14]. Similar approximations have been used by Stayman and Fessler [15] in designing a penalty function to achieve isotropic local impulse response functions. Combining with computer observer models, these theoretical results have been applied to the study of lesion detectability $[16-19]$. In $[17,19]$ the signalknown-exactly, background-known-exactly (SKE-BKE) detection task was used, which is a highly simplified scenario compared to real situations. While some variability of background and lesion was included in $[16,18]$, they both assumed that the covariances of the background and lesion are stationary (or at least locally stationary) for fast computation. Such a stationary assumption is generally invalid for random lesions. 
Here we study lesion detectability where both the lesion and background are described by statistical distributions. We derive simplified theoretical expressions that allow fast evaluation of lesion detectability for various linear observers without assuming the object covariance is locally stationary.

\section{Theory}

\subsection{Data Model}

Emission data are well modeled as a collection of independent Poisson random variables with the (conditional) expectation, $\overline{\boldsymbol{y}} \in \mathbb{R}^{M \times 1}$, related to the unknown tracer distribution, $\boldsymbol{x} \in \mathbb{R}^{N \times 1}$, through an affine transform

$$
\overline{\boldsymbol{y}} \equiv E[\boldsymbol{y} \mid \boldsymbol{x}]=\boldsymbol{P} \boldsymbol{x}+\boldsymbol{r},
$$

where $\boldsymbol{P} \in \mathbb{R}^{M \times N}$ is the detection probability matrix with the $(i, j)$ th element equal to the probability of detecting an event from the $j$ th voxel at the $i$ th measurement with consideration of photon attenuation and detector efficiency, and $\boldsymbol{r} \in \mathbb{R}^{M \times 1}$ accounts for the presence of scattered and random events in the data.

The Poisson likelihood function is

$$
p(\boldsymbol{y} \mid \boldsymbol{x})=\prod_{i} \frac{e^{-\bar{y}_{i}} \bar{y}_{i}^{y_{i}}}{y_{i} !},
$$

and the log likelihood function is given by

$$
L(\boldsymbol{y} \mid \boldsymbol{x})=\sum_{i}\left(y_{i} \log \bar{y}_{i}-\bar{y}_{i}-\log y_{i} !\right),
$$

where $\boldsymbol{y} \in \mathbb{R}^{M \times 1}$ is the measured sinogram data. For PET data that are precorrected for random events, a shifted Poisson model can be used [20].

\subsection{Bayesian Image Reconstruction}

Bayesian methods regularize the image through the use of a prior distribution on the unknown image. Most image priors have a Gibbs distribution of the form

$$
p(\boldsymbol{x})=\frac{1}{Z} e^{-\beta U(\boldsymbol{x})},
$$

where $U(\boldsymbol{x})$ is the energy function, $\beta$ is the smoothing parameter that controls the resolution of the reconstructed image, and $Z$ is a normalization constant. Here we focus on quadratic priors, for which the energy function can be expressed as

$$
U(\boldsymbol{x})=\frac{1}{2} \boldsymbol{x}^{\prime} \boldsymbol{R} \boldsymbol{x},
$$


where $\boldsymbol{R}$ is a positive definite (or semidefinite) matrix and ' denotes transpose. The commonly used pair-wise quadratic priors and thin plate priors [21,22] are just special cases of (5).

Combining the likelihood function and the image prior, the MAP reconstruction is found as

$$
\hat{\boldsymbol{x}}(\boldsymbol{y})=\arg \max _{\boldsymbol{x} \geq 0}[L(\boldsymbol{y} \mid \boldsymbol{x})-\beta U(\boldsymbol{x})] .
$$

Since $L(\boldsymbol{y} \mid \boldsymbol{x})$ is a concave function of $\boldsymbol{x},(6)$ generally has a unique solution for convex priors. The smoothing parameter $\beta$ has a strong effect on the image property. If $\beta$ is too small, the reconstructed image approaches the ML estimate and becomes very noisy; if $\beta$ is too large, the reconstructed image will be very smooth and useful structural information can be lost. The goal of this paper is to derive theoretical expressions for fast evaluation of the detectability of lesions in each data set with different reconstruction parameters.

\subsection{Lesion Detection with Numerical Observers}

For a given reconstructed image $\hat{\boldsymbol{x}}$, a linear numerical observer computes a test statistic (a scalar-valued decision variable), $\eta(\hat{\boldsymbol{x}})$, by

$$
\eta(\hat{\boldsymbol{x}})=\boldsymbol{t}^{\prime} \hat{\boldsymbol{x}}
$$

where $\boldsymbol{t}$ is the observer template. A decision whether there is a lesion or not is then made by comparing this statistic to a preselected threshold. If $\eta(\hat{\boldsymbol{x}})$ exceeds the decision threshold, $\hat{\boldsymbol{x}}$ is determined to have a lesion; otherwise, it is not. By varying the threshold, we can obtain a ROC curve. The area under the ROC curve (AUC) is often used to measure the lesion detectability. This numerical observer model assumes that the location of the possible lesion is known a priori. One can use separate observer templates for different locations.

The detection performance can also be measured by the SNR of $\eta(\hat{\boldsymbol{x}})$, which is defined as

$$
\begin{aligned}
\mathrm{SNR}^{2}[\eta(\hat{\boldsymbol{x}})] & =\frac{\left(E\left[\eta(\hat{\boldsymbol{x}}) \mid H_{1}\right]-E\left[\eta(\hat{\boldsymbol{x}}) \mid H_{0}\right]\right)^{2}}{\left(\operatorname{var}\left[\eta(\hat{\boldsymbol{x}}) \mid H_{1}\right]+\operatorname{var}\left[\eta(\hat{\boldsymbol{x}}) \mid H_{1}\right]\right) / 2}, \\
& =\frac{2\left(\boldsymbol{t}^{\prime} \boldsymbol{z}\right)^{2}}{\boldsymbol{t}^{\prime} \Sigma_{\hat{\boldsymbol{x}} \mid H_{1}} \boldsymbol{t}+\boldsymbol{t}^{\prime} \Sigma_{\hat{\boldsymbol{x}} \mid H_{0}} \boldsymbol{t}} .
\end{aligned}
$$

where $E$ denotes expectation, $H_{0}$ is the null hypothesis representing lesion absent, $H_{1}$ is the hypothesis representing lesion present, $\Sigma_{\hat{\boldsymbol{x}} \mid H_{1}}$ and $\Sigma_{\hat{\boldsymbol{x}} \mid H_{0}}$ are the covariance matrices of $\hat{\boldsymbol{x}}$ under hypotheses of $H_{1}$ and $H_{0}$, respectively, and $\boldsymbol{z} \equiv E\left[\hat{\boldsymbol{x}} \mid H_{1}\right]-E\left[\hat{\boldsymbol{x}} \mid H_{0}\right]$. Without loss of generality, we assume that the probabilities of the two hypotheses are equal.

When $\eta(\hat{\boldsymbol{x}})$ is normally distributed, the AUC is related to the SNR by [23]

$$
\mathrm{AUC}=\frac{1}{2}\left[1+\operatorname{erf}\left(\frac{\mathrm{SNR}}{2}\right)\right]
$$


where $\operatorname{erf}(x)$ is the error function. Here we will use SNR to measure the lesion detectability.

One example of the linear observers is the non-prewhitening (NPW) observer, which uses a matched filter to compute the test statistic [5]

$$
\eta_{\mathrm{NPW}}(\hat{\boldsymbol{x}})=\left(E\left[\hat{\boldsymbol{x}} \mid H_{1}\right]-E\left[\hat{\boldsymbol{x}} \mid H_{0}\right]\right)^{\prime} \hat{\boldsymbol{x}} \equiv \boldsymbol{z}^{\prime} \hat{\boldsymbol{x}} .
$$

In some situations, the NPW observer has been found to correlate with human performance for lesion detection [24-26].

At this time, the most popular numerical observers are probably the channelized Hotelling observers (CHOs) [27,25]. They have gained much interest because many studies have shown that $\mathrm{CHO}$ s have good correlation with human performance, although the degree of the correlation depends on the properties of the lesion and background, and the channel functions [28-30].

The test statistic of $\mathrm{CHO}$ is

$$
\eta_{C H O}(\hat{\boldsymbol{x}})=\boldsymbol{z}^{\prime} \boldsymbol{U}^{\prime} K^{-1} \boldsymbol{U} \hat{\boldsymbol{x}}
$$

where $\boldsymbol{U}$ denotes the frequency-selective channels that mimic the human visual system and $K$ is the covariance of the channel outputs, i.e.,

$$
K=\frac{1}{2} \boldsymbol{U}\left(\Sigma_{\hat{\boldsymbol{x}} \mid H_{1}}+\Sigma_{\hat{\boldsymbol{x}} \mid H_{0}}\right) \boldsymbol{U}^{\prime}+K_{N}
$$

where $K_{N}$ is the covariance of the internal noise in the channels to model the uncertainty in human detection process [31, 27].

\subsection{Lesion Detectability in MAP Reconstruction}

To compute the SNR for each numerical observer, we need the expressions of $\boldsymbol{z} \equiv E\left[\hat{\boldsymbol{x}} \mid H_{1}\right]-E\left[\hat{\boldsymbol{x}} \mid H_{0}\right]$ and covariance matrices $\Sigma_{\hat{\boldsymbol{x}} \mid H_{1}}$ and $\Sigma_{\hat{\boldsymbol{x}} \mid H_{0}}$. Using the results in [12] and [11], the local impulse response of MAP reconstruction can be approximated by

$$
\operatorname{LIR}_{\hat{\boldsymbol{x}}} \approx[\boldsymbol{F}+\beta \boldsymbol{R}]^{-1} \boldsymbol{F}
$$

and the covariance by

$$
\Sigma_{\hat{\boldsymbol{x}} \mid H_{k}} \approx[\boldsymbol{F}+\beta \boldsymbol{R}]^{-1} \boldsymbol{P}^{\prime} \operatorname{diag}\left[\frac{1}{\overline{\bar{y}}_{i}}\right] \Sigma_{y \mid H_{k}} \operatorname{diag}\left[\frac{1}{\overline{\bar{y}}_{i}}\right] \boldsymbol{P}[\boldsymbol{F}+\beta \boldsymbol{R}]^{-1} .
$$

where $\overline{\overline{\boldsymbol{y}}} \equiv \boldsymbol{P} E[\boldsymbol{x}]+\boldsymbol{r}=\frac{1}{2} \boldsymbol{P}\left(E\left[\boldsymbol{x} \mid H_{0}\right]+E\left[\boldsymbol{x} \mid H_{1}\right]\right)+\boldsymbol{r}, \boldsymbol{F} \equiv \boldsymbol{P}^{\prime} \operatorname{diag}\left[\frac{1}{\overline{\bar{y}}_{i}}\right] \boldsymbol{P}$, and $\Sigma_{y \mid H_{k}}$ is the covariance matrix of the measurement $\boldsymbol{y}$ under $H_{k}, k=0,1$. Note (13) represents the spatially variant local impulse response function with each column denoting the local impulse response at the corresponding voxel location.

Without object variability, $\boldsymbol{y}$ consists of independent Poisson random variables, of which the covariance is $\Sigma_{y \mid x}=\operatorname{diag}\left[\bar{y}_{i}\right]$ with $\overline{\boldsymbol{y}}=\boldsymbol{P} \boldsymbol{x}+\boldsymbol{r}$. When considering object variation, the overall covariance of the measurements is

$$
\Sigma_{y \mid H_{k}}=E\left\{\Sigma_{y \mid x} \mid H_{k}\right\}+\boldsymbol{P} \Sigma_{x \mid H_{k}} \boldsymbol{P}^{\prime}
$$


where $\Sigma_{x \mid H_{k}}$ is the covariance of the object variation under $H_{k}$.

Since we are particularly interested in small lesions, we can assumed that the presence of a lesion almost has no effect on the Poisson noise in the data, i.e., $E\left\{\Sigma_{y \mid x} \mid H_{k}\right\} \approx \operatorname{diag}\left[\overline{\bar{y}}_{i}\right], \quad k=0,1$. For small lesions, $\boldsymbol{z}$ can also be approximated by the convolution between the expectation of the lesion profile $\overline{\boldsymbol{f}}_{l} \equiv E\left[\boldsymbol{x} \mid H_{1}\right]-$ $E\left[\boldsymbol{x} \mid H_{0}\right]$ and the local impulse response function at the lesion location. Therefore, we have

$$
\begin{aligned}
\boldsymbol{z} & \approx[\boldsymbol{F}+\beta \boldsymbol{R}]^{-1} \boldsymbol{F} \overline{\boldsymbol{f}}_{l} \\
\Sigma_{\hat{\boldsymbol{x}} \mid H_{k}} & \approx[\boldsymbol{F}+\beta \boldsymbol{R}]^{-1}\left[\boldsymbol{F}+\boldsymbol{F} \Sigma_{x \mid H_{k}} \boldsymbol{F}\right][\boldsymbol{F}+\beta \boldsymbol{R}]^{-1} .
\end{aligned}
$$

Substituting (16) and (17) into (9), we can obtain the theoretical expressions of the SNR of any linear numerical observer in the form of (7). In particular, for $\mathrm{CHO}$ in (11), the SNR is

$$
\mathrm{SNR}_{C H O}^{2}=\boldsymbol{z}^{\prime} \boldsymbol{U}^{\prime} K^{-1} \boldsymbol{U} \boldsymbol{z},
$$

where

$$
K \approx \boldsymbol{U}[\boldsymbol{F}+\beta \boldsymbol{R}]^{-1}\left[\boldsymbol{F}+\boldsymbol{F} \Sigma_{x} \boldsymbol{F}\right][\boldsymbol{F}+\beta \boldsymbol{R}]^{-1} \boldsymbol{U}^{\prime}+K_{N},
$$

and $\Sigma_{x}$ is defined as $\frac{1}{2} \Sigma_{x \mid H_{0}}+\frac{1}{2} \Sigma_{x \mid H_{1}}$.

\subsection{Fast Computation}

Direct computation of (18) is very time-consuming due to the large size of the matrix. Often people assume the local impulse function and the covariance are locally stationary and use fast Fourier transform to compute the expressions in frequency domain (e.g., $[16,18])$. However, for random lesions that are independent of the background, the covariance of the object is not stationary around the lesion. This has been one major difficulty in computing the detectability of random lesions. Here we solve this problem by dividing the variance in (19) into two parts:

$$
\boldsymbol{U}[\boldsymbol{F}+\beta \boldsymbol{R}]^{-1} \boldsymbol{F}[\boldsymbol{F}+\beta \boldsymbol{R}]^{-1} \boldsymbol{U}^{\prime},
$$

which is caused by the Poisson noise in the data, and

$$
\boldsymbol{U}[\boldsymbol{F}+\beta \boldsymbol{R}]^{-1} \boldsymbol{F} \Sigma_{x} \boldsymbol{F}[\boldsymbol{F}+\beta \boldsymbol{R}]^{-1} \boldsymbol{U}^{\prime},
$$

which is due to the lesion (and background) variation.

Equation (20) is the same as the covariance matrix studies in $[16,18]$ and can be approximately computed using fast Fourier transform in frequency space based on the fact that $\boldsymbol{F}$ and $\boldsymbol{R}$ are locally stationary [14], i.e.,

$$
\boldsymbol{U}[\boldsymbol{F}+\beta \boldsymbol{R}]^{-1} \boldsymbol{F}[\boldsymbol{F}+\beta \boldsymbol{R}]^{-1} \boldsymbol{U}^{\prime} \approx \tilde{\boldsymbol{U}} \operatorname{diag}\left[\frac{\lambda_{i}}{\left(\lambda_{i}+\beta \mu_{i}\right)^{2}}\right] \tilde{\boldsymbol{U}}^{\prime},
$$

where $\left\{\lambda_{i}, i=1, \ldots, N\right\}$ and $\left\{\mu_{i}, i=1, \ldots, N\right\}$ are the Fourier coefficients of the column vectors corresponding to the lesion location of $\boldsymbol{F}$ and $\boldsymbol{R}$, respectively, and $\tilde{\boldsymbol{U}}$ is the Fourier coefficients of the channel functions. Details on the computation of $\boldsymbol{\lambda}$ and $\boldsymbol{\mu}$ can be found in [14]. 
Since $\boldsymbol{x}$ is not stationary, $\Sigma_{x}$ is not a block Toeplitz matrix and hence equation (21) cannot be computed in frequency space. However, we found that we can compute $\boldsymbol{F}[\boldsymbol{F}+\beta \boldsymbol{R}]^{-1} \boldsymbol{U}^{\prime}$ in Fourier space and then calculate the product with $\Sigma_{x}$ in spatial domain, Thus (21) can be approximated by

$$
\begin{aligned}
& \boldsymbol{U}[\boldsymbol{F}+\beta \boldsymbol{R}]^{-1} \boldsymbol{F} \Sigma_{x} \boldsymbol{F}[\boldsymbol{F}+\beta \boldsymbol{R}]^{-1} \boldsymbol{U}^{\prime} \\
\approx & \left(\boldsymbol{Q}^{\prime} \operatorname{diag}\left[\frac{\lambda_{i}}{\lambda_{i}+\beta \mu_{i}}\right] \tilde{\boldsymbol{U}}^{\prime}\right)^{\prime} \Sigma_{x}\left(\boldsymbol{Q}^{\prime} \operatorname{diag}\left[\frac{\lambda_{i}}{\lambda_{i}+\beta \mu_{i}}\right] \tilde{\boldsymbol{U}}^{\prime}\right),
\end{aligned}
$$

where $\boldsymbol{Q}$ represents the Kronecker form of the Fourier transform. Because the number of channels is small (often less than 10), (23) can be computed very easily. Since we have used the locally stationary approximation on $\boldsymbol{F}[\boldsymbol{F}+\beta \boldsymbol{R}]^{-1},(23)$ requires that the correlation length in $\Sigma_{x}$ is relatively short and that the energy of the channel function $\boldsymbol{U}$ concentrates around the lesion location.

Using similar approximations, $\boldsymbol{z}$ can be computed in the Fourier space as

$$
\boldsymbol{U} \boldsymbol{z}=\tilde{\boldsymbol{U}} \operatorname{diag}\left[\frac{\lambda_{i}}{\lambda_{i}+\beta \mu_{i}}\right] \boldsymbol{\xi}=\left[\sum_{i} \frac{\tilde{U}_{k, i} \lambda_{i} \xi_{i}}{\lambda_{i}+\beta \mu_{i}}\right],
$$

where $\left\{\xi_{i}, i=1, \ldots, N\right\}$ is the Fourier transforms of $\overline{\boldsymbol{f}}_{l}$, and $\left[c_{k}\right]$ denotes a column vector with the $k$ th element being $c_{k}$.

Substituting (22)-(24) into (18), we can get the final expressions for SNR of $\mathrm{CHO}$

$$
\mathrm{SNR}_{C H O}^{2}=\left[\sum_{i} \frac{\tilde{U}_{k, i} \lambda_{i} \xi_{i}}{\lambda_{i}+\beta \mu_{i}}\right]^{\prime} K^{-1}\left[\sum_{i} \frac{\tilde{U}_{k, i} \lambda_{i} \xi_{i}}{\lambda_{i}+\beta \mu_{i}}\right],
$$

where

$$
\begin{aligned}
K \approx \tilde{\boldsymbol{U}} & \operatorname{diag}\left[\frac{\lambda_{i}}{\left(\lambda_{i}+\beta \mu_{i}\right)^{2}}\right] \tilde{\boldsymbol{U}}^{\prime} \\
& +\left(\boldsymbol{Q}^{\prime} \operatorname{diag}\left[\frac{\lambda_{i}}{\lambda_{i}+\beta \mu_{i}}\right] \tilde{\boldsymbol{U}}^{\prime}\right)^{\prime} \Sigma_{x}\left(\boldsymbol{Q}^{\prime} \operatorname{diag}\left[\frac{\lambda_{i}}{\lambda_{i}+\beta \mu_{i}}\right] \tilde{\boldsymbol{U}}^{\prime}\right)+K_{N} .
\end{aligned}
$$

\subsection{Lesion with Known Profile}

As a special case, we study the random lesions with known profile. Here the tracer uptake in lesions is modeled as a fixed profile times a random scaling factor $c$ with mean of one and variance of $\sigma_{c}^{2}$ (variable contrast), i.e.,

$$
\boldsymbol{f}_{l}=c \overline{\boldsymbol{f}}_{l} \text {. }
$$

This statistical model is especially useful in emission imaging when the tracer uptake in small lesions can be assumed to be homogeneous. The covariance of the lesion under this model is

$$
\Sigma_{\boldsymbol{f}_{l}}=\sigma_{c}^{2} \overline{\boldsymbol{f}}_{l} \overline{\boldsymbol{f}}_{l}^{\prime}
$$




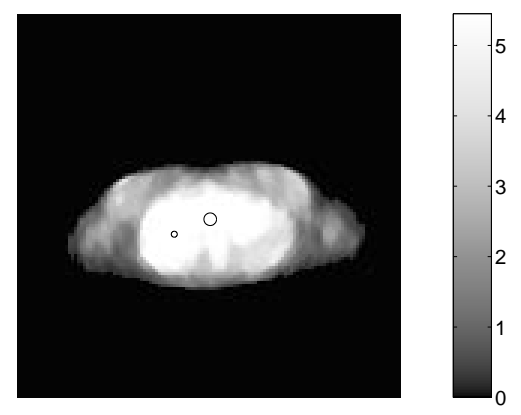

Fig. 1. The background phantom image and the locations of the simulated lesions.

Substituting it into (21) and assuming there is no background variation, we get

$$
\boldsymbol{U}[\boldsymbol{F}+\beta \boldsymbol{R}]^{-1} \boldsymbol{F} \Sigma_{x} \boldsymbol{F}[\boldsymbol{F}+\beta \boldsymbol{R}]^{-1} \boldsymbol{U}^{\prime}=\sigma_{c}^{2} \boldsymbol{U} \boldsymbol{z} \boldsymbol{z}^{\prime} \boldsymbol{U}^{\prime},
$$

and the covariance of channel output $K$ becomes

$$
K \approx \tilde{\boldsymbol{U}} \operatorname{diag}\left[\frac{\lambda_{i}}{\left(\lambda_{i}+\beta \mu_{i}\right)^{2}}\right] \tilde{\boldsymbol{U}}^{\prime}+\sigma_{c}^{2}\left[\sum_{i} \frac{\tilde{U}_{k, i} \lambda_{i} \xi_{i}}{\lambda_{i}+\beta \mu_{i}}\right]\left[\sum_{i} \frac{\tilde{U}_{k, i} \lambda_{i} \xi_{i}}{\lambda_{i}+\beta \mu_{i}}\right]^{\prime}+K_{N},
$$

which can be computed in frequency space without assuming $\Sigma_{x}$ is locally stationary!

\section{Monte Carlo Simulations}

We conducted computer simulations to validate our theoretical approximations. We simulated an ECAT HR+ whole-body PET scanner (CTI PET Systems, Knoxville, TN) operating in two dimension mode. The sinogram data has 288 angles of view and 288 lines of response in each view. The background phantom image (Figure 1) was obtained from a reconstructed image of a patient scan. Two circular lesions with variable contrast, one $8 \mathrm{~mm}$ diameter and one $16 \mathrm{~mm}$ diameter, were simulated in the liver region. The locations are shown in Figure 1.

The data were generated by forward projecting the phantom image with and without a lesion. Photon attenuation was modeled. Poisson noise was added to the sinogram data after scaling the expected total number of events to 200k. For each case, 500 independent noisy data sets were reconstructed using a preconditioned conjugated gradient method [32]. The SNRs for the CHO were calculated using both Monte Carlo reconstructions and the theoretical expressions with (25) and (30). Two sets of channel functions were studied: (i) five rotationally symmetric, non-overlapping square channels (SQR); and (ii) three difference-ofGaussian channels (DOG). These channel functions are similar to that used in $[25,33]$. 


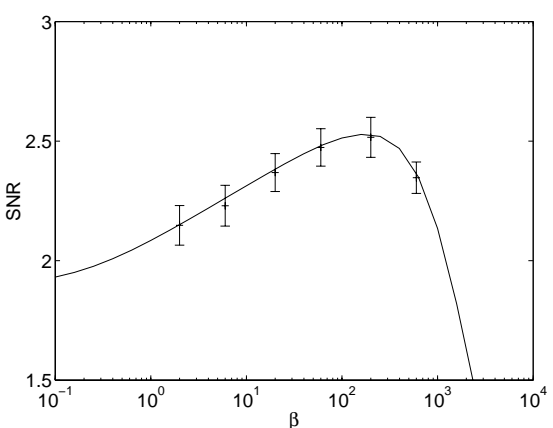

(a)

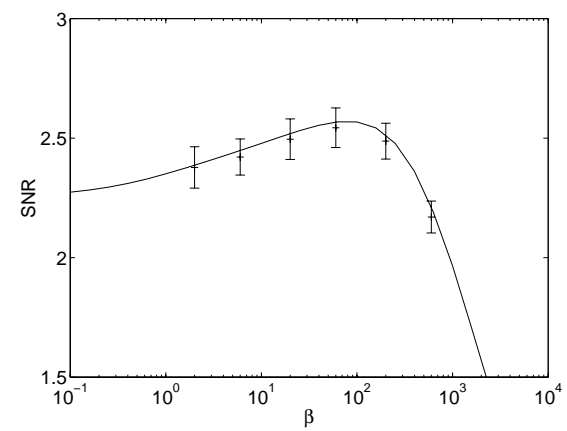

(c)

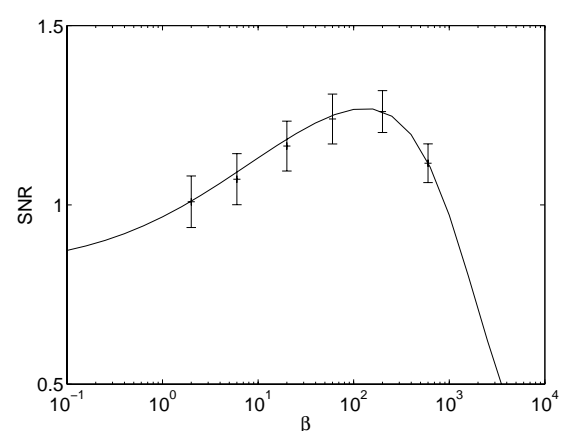

(b)

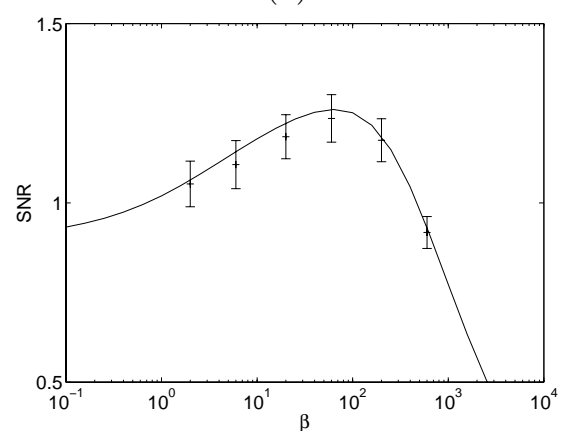

(d)

Fig. 2. Comparison of the Monte Carlo results ('+') with the theoretical predications (solid line). (a) $16 \mathrm{~mm}$ lesion with DOG channels; (b) $8 \mathrm{~mm}$ lesion with DOG channels; (c) $16 \mathrm{~mm}$ lesion with SQR channels; (d) $8 \mathrm{~mm}$ lesion with SQR channels. The tumor to background activity ratio was uniformly distributed between 1.18 and 1.75 .

Figure 2 shows the SNRs computed from independent Monte Carlo reconstructions and the theoretical approximations. The tumor to background activity ratio was uniformly distributed between 1.18 and 1.75 . The background activity was fixed with the maximum at 5.5. White noise with variance of $5 \times 10^{-3}$ was used to model the internal channel noise in human visual system. The error bars in the Monte Carlo results represent $68 \%$ confidence intervals that were estimated using a bootstrap method. In general, the theoretical predictions match with Monte Carlo results very well. All theoretical predictions lie inside the $68 \%$ confidence interval of the Monte Carlo estimates. We can also see that the optimum smoothing parameter (maximum SNR) for the $16 \mathrm{~mm}$ lesion is slightly larger than that for the $8 \mathrm{~mm}$ lesion, indicating that lower resolution image is preferred for detecting larger lesions.

Figure 3 shows another comparison of the CHO performance for the $8 \mathrm{~mm}$ lesion with activity ratio varying from 1.75 to 3.2 . With the higher contrast, the lesion detectability is significantly increased, but the shape of the curves 


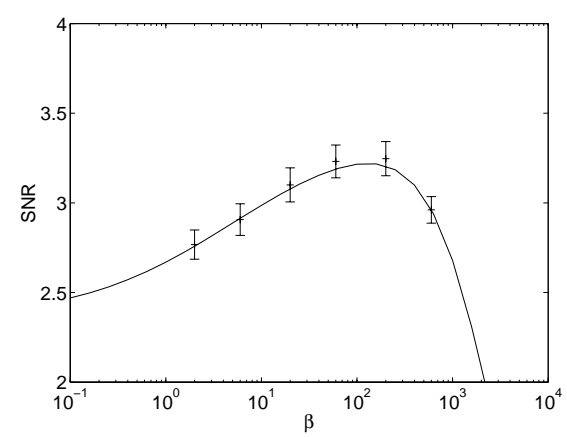

(a)

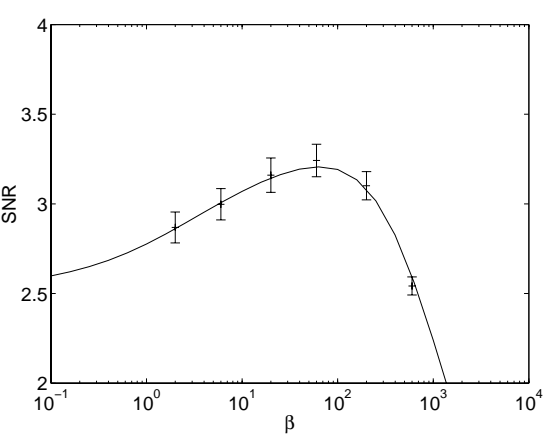

(b)

Fig. 3. Comparison of the Monte Carlo results ('+') with the theoretical predications (solid line) for the $8 \mathrm{~mm}$ lesion. (a) DOG channels; (b) SQR channels. The tumor to background activity ratio was uniformly distributed between 1.75 and 3.2.

are similar to those shown in Figure 2. Again, good match between theoretical predictions and Monte Carlo results is evident. Additional simulations show that the lesion detectability can also be reliably estimated by the theoretical expressions using noisy data.

\section{Conclusion and Discussion}

We have derived theoretical expressions for fast computation of lesion detectability in the Bayesian reconstruction. Both lesion and the background can contain variability. No assumption on the local stationary of the object variation is used. The results are applicable to a wide range of linear numerical observers and can be used to find the optimum regularization for lesion detection. We have conducted Monte Carlo simulations and the comparisons show good agreement between the theoretical predications and the Monte Carlo results.

In our experiments (results not shown) we have found that the lesion detectability depends on the channel parameters of the observer. In particular, the lesion detectability with large $\beta(>100)$ heavily depends on the internal noise level in the low frequency channels. Finding the best channel parameters that correlated with human performance is essential for optimizing image reconstruction for lesion detection. Research has already been conducted to estimate the observer template directly from human-observer studies (e.g.,[34]). While the channel parameters used in this paper are somewhat arbitrary and are unlikely to be the optimum, the theoretical results do not rely on the particular choice of the channel parameters and can be applied to almost any channel parameters that are suitable for the detection task.

Another issue is that the Bayesian reconstruction algorithm that we studied uses a Gibbs prior with a quadratic energy function. The statistical information about the lesion and the background is only used in the observer study. We 
choose this simple form of Bayesian method because it is widely used in practice. In a true Bayesian paradigm, however, one may want to use all the prior information in the reconstruction process (including the distribution of the lesion and background). It would be interesting to see how such a true Bayesian approach will affect the image quality. This is one of our future research directions.

\section{Acknowledgments}

The author would like to thank Drs. Richard Leahy, Ronald Huesman, and Grant Gullberg for various discussions on this topic, and thank Dr. Jeffery Fessler and anonymous reviewers for their valuable comments on the manuscript.

\section{References}

1. Fessler, J.A.: Penalized weighted least squares image reconstruction for PET. IEEE Trans. Med. Im. 13 (1994) 290-300

2. Mumcuoglu, E., Leahy, R., Cherry, S., Zhou, Z.: Fast gradient-based methods for Bayesian reconstruction of transmission and emission PET images. IEEE Trans Med Im 13 (1994) 687-701

3. Fessler, J.A., Hero, A.O.: Penalized maximum-likelihood image reconstruction using space-alternating generalized EM algorithms. IEEE Trans Im Proc 4 (1995) 1417-1429

4. Bouman, C., Sauer, K.: A unified approach to statistical tomography using coordinate descent optimization. IEEE Trans Im Proc 5 (1996) 480-492

5. Barrett, H.H., Yao, J., Rolland, J., Myers, K.: Model observers for assessment of image quality. Proc. Natl. Acad. Sci. 90 (1993) 9758-9765

6. Barrett, H.H., Wilson, D.W., Tsui, B.M.W.: Noise properties of the EM algorithm: I. theory. Phy. Med. Bio. 39 (1994) 833-846

7. Wang, W., Gindi, G.: Noise analysis of MAP-EM algorithms for emission tomography. Phy. Med. Bio. 42 (1997) 2215-2232

8. Soares, E.J., Byrne, C., Glick, S.: Noise characterization of block-iterative reconstruction algorithms: 1. theory. IEEE Trans Med Im 19 (2000) 261-270

9. Abbey, C.K., Barrett, H.H.: Observer signal-to-noise ratios for the ML-EM algorithm. In: Proceedings of SPIE. Volume 2712. (1996) 47-58

10. Hudson, H.M., Larkin, R.S.: Accelerated image reconstruction using ordered subsets of projection data. IEEE Trans Med Im 13 (1994) 601-609

11. Fessler, J.: Mean and variance of implicitely defined biased estimators (such as penalized maximum likelihood): Applications to tomography. IEEE Trans Im Proc 5 (1996) 493-506

12. Fessler, J.A., Rogers, W.L.: Spatial resolution properties of penalized-likelihood image reconstruction: Spatial-invariant tomographs. IEEE Trans Im Proc 9 (1996) 1346-1358

13. Qi, J., Leahy, R.M.: A theoretical study of the contrast recovery and variance of MAP reconstructions from PET data. IEEE Trans Med Im 18 (1999) 293-305

14. Qi, J., Leahy, R.M.: Resolution and noise properties of MAP reconstruction for fully 3D PET. IEEE Trans Med Im 19 (2000) 493-506 
15. Stayman, J.W., Fessler, J.A.: Regularization for uniform spatial resolution properties in penalized-likelihood image reconstruction. IEEE Trans Med Im 19 (2000) 601-615

16. Bonetto, P., Qi, J., Leahy, R.M.: Covariance approximation for fast and accurate computation of channelized Hotelling observer statistics. IEEE Trans. Nucl. Sci. 47 (2000) 1567-1572

17. Qi, J., Huesman, R.H.: Theoretical study of lesion detectability of MAP reconstruction using computer observers. IEEE Trans Med Im 20 (2001) 815-822

18. Fessler, J.A., Yendiki, A.: Channelized Hotelling observer performance for penalized-likelihood image reconstruction. In: Proc. IEEE NSS-MIC. (2002) to appear

19. Xing, Y., Gindi, G.: Rapid calculation of detectability in Bayesian SPECT. In: Proceedings of IEEE International Symposimum on Biomedical Imaging. (2002) CDROM

20. Yavuz, M., Fessler, J.A.: Statistical image reconstruction methods for randomsprecorrected PET scans. Medical Image Analysis 2 (1998) 369-378

21. Blake, A., Zisserman, A.: Visual Reconstruction. The MIT Press (1987)

22. Lee, S.J., Rangarajan, A., Gindi, G.: Bayesian image reconstruction in SPECT using higher oreder mechanical models as priors. IEEE Trans Med Im 14 (1995) $669-680$

23. Barrett, H.H., Gooley, T., Girodias, K., Rolland, J., White, T., Yao, J.: Linear discriminants and image quality. Image and Vision Computing 10 (1992) 451-460

24. Myers, K.J., Barrett, H.H., Borgstrom, M.C., Patton, D.D., Seeley, G.W.: Effect of noise correlation on detectability of disk signals in medical imaging. Journal of the Optical Society of America A 2 (1985) 1752-1759

25. Myers, K.J., Barrett, H.H.: Addition of a channel mechanism to the ideal-observer model. Journal of the Optical Society of America A 4 (1987) 2447-2457

26. de Vries, D., King, M., Soares, E., Tsui, B., Metz, C.: Effects of scatter subtraction on detection and quantitation in hepatic SPECT. J. Nucl. Med. 40 (1999) 10111023

27. Yao, J., Barrett, H.H.: Predicting human performance by a channelized Hotelling model. In: Proc. of SPIE. 1768 (1992) 161-168

28. Abbey, C.K., Barrett, H.H.: Observer signal-to-noise ratios for the ML-EM algorithm. In: Proc. of SPIE. 2712 (1996) 47-58

29. Narayan, T., Herman, G.: Prediction of human observer performance by numerical observers: an experimental study. Journal of Optical Society of America A 16 (1999) 679-693

30. Gifford, H., King, M., de Vries, D., Soares, E.: Channelized hotelling and human observer correlation for lesion detection in hepatic SPECT imaging. J. Nucl. Med. 41 (2000) 514-521

31. Burgess, A.E., Colborne, E.: Visual signal detection. IV. obserer inconsistency. Journal of Optical Society of America A 5 (1988) 617-627

32. Qi, J., Leahy, R.M., Cherry, S.R., Chatziioannou, A., Farquhar, T.H.: High resolution 3D Bayesian image reconstruction using the microPET small animal scanner. Phy. Med. Bio. 43 (1998) 1001-1013

33. King, M., de Vries, D., Soares, E.: Comparison of the channelised hotelling and human observers for lesion detection in hepatic SPECT imaging. In: Proc. of SPIE 3036 (1997) 14-20

34. Abbey, C.K., Eckstein, M.P.: Optimal shifted estimates of human-observer templates in two-alternative forced-choice experiments. IEEE Trans Med Im 21 (2002) 429-440 